\title{
Positive solutions to nonlinear singular second order boundary value problems
}

\author{
by Gabriele Bonanno (Reggio Calabria)
}

\begin{abstract}
Existence theorems of positive solutions to a class of singular second order boundary value problems of the form $y^{\prime \prime}+f\left(x, y, y^{\prime}\right)=0,0<x<1$, are established. It is not required that the function $(x, y, z) \rightarrow f(x, y, z)$ be nonincreasing in $y$ and/or $z$, as is generally assumed. However, when $(x, y, z) \rightarrow f(x, y, z)$ is nonincreasing in $y$ and $z$, some of O'Regan's results [J. Differential Equations 84 (1990), 228-251] are improved. The proofs of the main theorems are based on a fixed point theorem for weakly sequentially continuous operators.
\end{abstract}

1. Introduction. The study of positive solutions to singular nonlinear boundary value problems applied to specific models arising from mathematical physics $[5,8,11]$ has also acquired theoretical interest in the past few years $[2,3,7,9,10]$. The works in this field of research are strictly based on the fundamental hypothesis of the function being nonincreasing with respect to dependent variables; given a second order ordinary differential equation $y^{\prime \prime}+f\left(x, y, y^{\prime}\right)=0$ with an appropriate boundary value, it is generally assumed that the function $(x, y, z) \rightarrow f(x, y, z)$ is nonincreasing in $y$ and/or $z$. Hence, the singularities at $y=0$ and at $z=0$ are imposed by the conditions $\lim _{y \rightarrow 0^{+}} f(x, y, z)=\infty$ for every $x$ and $z$, and $\lim _{z \rightarrow 0^{+}} f(x, y, z)$ $=\infty$ for every $x$ and $y$.

The aim of this paper is twofold: on the one hand, to present some existence theorems that do not necessarily require the above assumptions, and on the other hand, when these assumptions are employed, to improve some results already known. In the theorems presented here the above limits for $f$ may not necessarily exist, contrary to what is assumed, for instance, in [7]; however, when it is assumed that $f$ is nonincreasing, some results similar to those in [10] are obtained.

1991 Mathematics Subject Classification: Primary 34B15.

Key words and phrases: singular boundary value problem, positive solution. 
The work is divided into three parts. In the first part the fixed point theorem (Theorem 2.1) for weakly sequentially continuous operators proved in [1] is repeated. In the second part, the main results of the paper (Theorems 3.1-3.3) are given and two examples of how these theorems can be presented in a more practical way are shown (Theorems $3.4,3.5$ ). In the last part, some special cases of the main results are examined when it is assumed that $f$ is nonincreasing, and some of the results of [10] are improved.

2. Preliminaries and notations. Throughout this paper $L^{p}([0,1])$, $p \in[1, \infty[$, is the space of (equivalence classes of) measurable functions $\psi:[0,1] \rightarrow \mathbb{R}$ such that $\int_{0}^{1}|\psi(x)|^{p} d x<\infty$, equipped with the norm $\|\psi\|_{p}=\left(\int_{0}^{1}|\psi(x)|^{p} d x\right)^{1 / p}$. The symbol $W^{2,1}([0,1])$ indicates the set of all $u \in$ $C^{1}([0,1])$ such that $u^{\prime}$ is absolutely continuous in $[0,1]$ and $u^{\prime \prime} \in L^{1}([0,1])$. Obviously, "measurable" means Lebesgue measurable and $\mathbb{R}$ is the real Euclidean space.

Given a real function $f$, consider the problems

$$
\begin{gathered}
\left(\mathrm{P}_{1}\right)\left\{\begin{array} { l } 
{ y ^ { \prime \prime } + f ( x , y , y ^ { \prime } ) = 0 , } \\
{ y ( 0 ) = a \geq 0 , } \\
{ y ^ { \prime } ( 1 ) = b \geq 0 , }
\end{array} \quad ( \mathrm { P } _ { 2 } ) \left\{\begin{array}{l}
y^{\prime \prime}+f\left(x, y, y^{\prime}\right)=0, \\
y(0)=a \geq 0, \\
y(1)=b \geq 0,
\end{array}\right.\right. \\
\left(\mathrm{P}_{3}\right)\left\{\begin{array}{l}
y^{\prime \prime}+f\left(x, y, y^{\prime}\right)=0, \\
y(1)=a \geq 0, \\
y^{\prime}(0)=b \leq 0 .
\end{array}\right.
\end{gathered}
$$

A function $u:[0,1] \rightarrow \mathbb{R}$ is said to be a generalized solution to problem $\left(\mathrm{P}_{1}\right)$ [resp. $\left(\mathrm{P}_{2}\right)$ or $\left.\left(\mathrm{P}_{3}\right)\right]$ if $u \in W^{2,1}([0,1]), u(0)=a, u^{\prime}(1)=b$ [resp. $u(0)=a, u(1)=b$ or $\left.u(1)=a, u^{\prime}(0)=b\right]$ and $u^{\prime \prime}(x)=f\left(x, u(x), u^{\prime}(x)\right)$ for almost every $x \in[0,1]$. When $u(x)>0$ for every $x \in(0,1), u$ is said to be a positive solution.

The proof of our main results is based on the fixed point theorem for weakly sequentially continuous mappings proved in [1]. For the reader's convenience, we repeat it here.

Theorem 2.1 ([1, Theorem 1]). Let $X$ be a metrizable locally convex topological vector space and let $K$ be a weakly compact convex subset of $X$. Suppose $G$ is a weakly sequentially continuous map from $K$ into itself. Then there exists $v_{0} \in K$ such that $v_{0}=G\left(v_{0}\right)$.

In the sequel we will use the following notations. Let $\eta:[0,1] \rightarrow \mathbb{R}$ be an integrable function such that $\eta(x) \geq 0$ for almost every $x \in(0,1)$ and let $r>\|\eta\|_{1} \geq 0$. 
For every $x \in(0,1)$ we put

$$
\begin{aligned}
V \equiv V(\eta, r, x)=\{ & (y, z) \in(0, \infty) \times(0, \infty): \\
& \int_{0}^{x} s \eta(s) d s+x \int_{x}^{1} \eta(s) d s+b x+a \leq y \leq r x+b x+a ; \\
& \left.\int_{0}^{x} \eta(s) d s+b \leq z \leq b+r\right\},
\end{aligned}
$$

and

$$
m_{\eta, r}(x)=\inf _{V} f(x, \cdot, \cdot), \quad M_{\eta, r}(x)=\sup _{V} f(x, \cdot, \cdot),
$$

where $f$ is a real function defined in $[0,1] \times(0, \infty) \times(0, \infty)$.

Moreover, we put

$$
\begin{aligned}
T \equiv T(\eta, r, x)=\{ & (y, z) \in(0, \infty) \times(-\infty, \infty) \backslash\{0\}: \\
& (1-x) \int_{0}^{x} s \eta(s) d s+x \int_{x}^{1}(1-s) \eta(s) d s \\
& +(b-a) x+a \leq y \leq r x(1-x)+(b-a) x+a ; \\
& \left.\int_{x}^{1} \eta(s) d s+\int_{0}^{1} s \eta(s) d s+(b-a) \leq z \leq 2 r+b-a\right\}
\end{aligned}
$$

and

$$
l_{\eta, r}(x)=\inf _{T} f(x, \cdot, \cdot), \quad L_{\eta, r}(x)=\sup _{T} f(x, \cdot, \cdot),
$$

where $f$ is a real function defined in $[0,1] \times(0, \infty) \times(-\infty, \infty) \backslash\{0\}$.

Finally, we put

$$
\begin{aligned}
S \equiv S(\eta, r, x)=\{ & (y, z) \in(0, \infty) \times(-\infty, 0): \\
& (1-x) \int_{0}^{x} \eta(s) d s+\int_{x}^{1}(1-s) \eta(s) d s+b x+a-b \leq y \\
\leq & r(1-x)+b x+a-b ; \\
& \left.\int_{0}^{x} \eta(s) d s-b \leq-z \leq-b+r\right\}
\end{aligned}
$$

and

$$
e_{\eta, r}(x)=\inf _{S} f(x, \cdot, \cdot), \quad E_{\eta, r}(x)=\sup _{S} f(x, \cdot, \cdot),
$$

where $f$ is a real function defined in $[0,1] \times(0, \infty) \times(-\infty, 0)$. 
3. Main results. In this section we establish our main results: Theorems 3.1-3.3. In addition, we give two examples of how these theorems can be presented in a more practical way.

Theorem 3.1. Let $f:[0,1] \times(0, \infty) \times(0, \infty) \rightarrow \mathbb{R}$. Assume that

(a) $x \rightarrow f(x, y, z)$ is measurable for every $(y, z) \in(0, \infty) \times(0, \infty)$;

(b) $(y, z) \rightarrow f(x, y, z)$ is continuous for almost every $x \in(0,1)$;

(c) there exists a function $\eta:[0,1] \rightarrow \mathbb{R}$, with $\eta(x) \geq 0$ for almost every $x \in(0,1)$, and a constant $r>0$ such that

$\left(c_{1}\right) m_{\eta, r}(x)>\eta(x)$ for almost every $x \in(0,1)$;

(c, $M_{\eta, r} \in L^{1}([0,1])$;

$\left(\mathrm{c}_{3}\right)\left\|M_{\eta, r}\right\|_{1} \leq r$.

Then problem $\left(\mathrm{P}_{1}\right)$ admits at least one generalized solution $u \in W^{2,1}([0,1])$ such that $u(x)>0$ for every $x \in(0,1]$.

Proof. Consider the set

$$
K_{1}=\left\{v \in L^{1}([0,1]): m_{\eta, r}(x) \leq v(x) \leq M_{\eta, r}(x) \text { a.e. } x \text { in }(0,1)\right\} .
$$

Clearly, $K$ is a nonempty, closed, convex and bounded subset of $L^{1}([0,1])$. Moreover, by the Dunford-Pettis theorem (see, for instance, [6, Theorem 1, p. 101]) it is also weakly compact. Observe that, if $v \in K_{1}$, then, by $\left(\mathrm{c}_{3}\right)$,

$$
\|v\|_{1} \leq\left\|M_{\eta, r}\right\|_{1} \leq r .
$$

For $v \in L^{1}([0,1])$ and $x \in[0,1]$, we put

$$
\Phi_{1}(v)(x)=\int_{0}^{x} s v(s) d s+x \int_{x}^{1} v(s) d s+b x+a .
$$

Obviously, one has

$$
\Phi_{1}(v)^{\prime}(x)=\int_{x}^{1} v(s) d s+b
$$

and $\Phi_{1}(v)^{\prime \prime}(x)=-v(x)$ for every $x \in[0,1]$; further, $\Phi_{1}(v)(0)=a$ and $\Phi_{1}(v)^{\prime}(1)=b$. Hence, $\Phi_{1}(v) \in W^{2,1}([0,1])$. We now put

$$
G_{1}(v)(x)=f\left(x, \Phi_{1}(v)(x), \Phi_{1}(v)^{\prime}(x)\right)
$$

for $v \in L^{1}([0,1])$ and $x \in(0,1)$. We claim that $G_{1}\left(K_{1}\right) \subseteq K_{1}$. Indeed, pick $v \in K_{1}$ and observe that, by $\left(\mathrm{c}_{1}\right)$ and (3.1)-(3.3), we obtain

$$
\begin{aligned}
\int_{0}^{x} s \eta(s) d s+x \int_{x}^{1} s \eta(s) d s+b x+a \leq & \Phi_{1}(v)(x) \\
& \leq x \int_{0}^{1} v(s) d s+b x+a \leq r x+b x+a,
\end{aligned}
$$




$$
\int_{x}^{1} \eta(s) d s+b \leq \Phi_{1}(v)^{\prime}(x) \leq \int_{0}^{1} v(s) d s+b \leq r+b .
$$

Therefore, $\left(\Phi_{1}(v)(x), \Phi_{1}(v)^{\prime}(x)\right) \in V(\eta, r, x)$ for every $x \in(0,1)$. Hence,

$$
m_{\eta, r}(x) \leq f\left(x, \Phi_{1}(v)(x), \Phi_{1}(v)^{\prime}(x)\right) \leq M_{\eta, r}(x)
$$

for almost every $x \in(0,1)$ and our statement is proved.

Now, let us prove that the operator $G_{1}$ is weakly sequentially continuous. Fix $v \in K_{1}$ and let $\left\{v_{n}\right\}$ be a sequence in $K_{1}$ weakly converging to $v$ in $L^{1}([0,1])$. From (3.2) and (3.3) it follows that $\lim _{n \rightarrow \infty} \Phi_{1}\left(v_{n}\right)(x)=\Phi_{1}(v)(x)$ and $\lim _{n \rightarrow \infty} \Phi_{1}\left(v_{n}\right)^{\prime}(x)=\Phi_{1}(v)^{\prime}(x)$ for every $x \in(0,1)$. Therefore, by (b), the sequence $\left\{G_{1}\left(v_{n}\right)\right\}$ converges almost everywhere in $[0,1]$ to $G_{1}(v)$. Since

$$
\left|G\left(v_{n}\right)(x)\right| \leq M_{\eta, r}(x)
$$

for almost every $x \in[0,1]$ and every $n \in \mathbb{N}$, by the Lebesgue Dominated Convergence Theorem one has $\lim _{n \rightarrow \infty} G\left(v_{n}\right)=G(v)$ in $L^{1}([0,1])$. So, $\left\{G\left(v_{n}\right)\right\}$ converges weakly to $G(v)$ in $L^{1}([0,1])$. Now, Theorem 2.1 shows that there exists $v_{0} \in K_{1}$ such that $v_{0}=G_{1}\left(v_{0}\right)$. The function $u_{0}(x)=\Phi_{1}\left(v_{0}\right)(x)$, $x \in[0,1]$, satisfies our conclusion.

Theorem 3.2. Let $f:[0,1] \times(0, \infty) \times(-\infty, \infty) \backslash\{0\} \rightarrow \mathbb{R}$. Assume that

(a) $x \rightarrow f(x, y, z)$ is measurable for every $(y, z) \in(0, \infty) \times(-\infty, \infty) \backslash\{0\}$;

(b) $(y, z) \rightarrow f(x, y, z)$ is continuous for almost every $x \in(0,1)$;

(c) there exists a function $\eta:[0,1] \rightarrow \mathbb{R}$, with $\eta(x) \geq 0$ for almost every $x \in(0,1)$, and a constant $r>0$ such that

$$
\begin{aligned}
& \left(\mathrm{c}_{1}\right) l_{\eta, r}(x)>\eta(x) \text { for almost every } x \in(0,1) ; \\
& \left(\mathrm{c}_{2}\right) L_{\eta, r} \in L^{1}([0,1]) ; \\
& \left(\mathrm{c}_{3}\right)\left\|L_{\eta, r}\right\|_{1} \leq r .
\end{aligned}
$$

Then problem $\left(\mathrm{P}_{2}\right)$ admits at least one generalized solution $u \in W^{2,1}([0,1])$ such that $u(x)>0$ for every $x \in(0,1)$.

Proof. The proof is similar to that of Theorem 3.1 and so we only give a sketch. We define

$$
K_{2}=\left\{v \in L^{1}([0,1]): l_{\eta, r}(x) \leq v(x) \leq L_{\eta, r}(x) \text { a.e. } x \text { in }(0,1)\right\}
$$

and put

$$
\Phi_{2}(v)(x)=(1-x) \int_{0}^{x} s v(s) d s+x \int_{x}^{1}(1-s) v(s) d s+(b-a) x+a
$$

for $v \in L^{1}([0,1])$ and $x \in[0,1]$. Obviously, one has

$$
\Phi_{2}(v)^{\prime}(x)=\int_{x}^{1} v(s) d s+\int_{0}^{1} s v(s) d s+(b-a)
$$


and $\Phi_{2}(v)^{\prime \prime}(x)=-v(x)$ for every $x \in[0,1]$. Further, $\Phi_{2}(v)(0)=a$ and $\Phi_{2}(v)(1)=b$.

Moreover, we set $G_{2}(v)(x)=f\left(x, \Phi_{2}(v)(x), \Phi_{2}(v)^{\prime}(x)\right)$ for $v \in L^{1}([0,1])$ and $x \in(0,1)$. In the same way as in the proof of Theorem 3.1 it is proved that $G_{2}$ is a weakly sequentially continuous operator from $K_{2}$ into itself. Then, by Theorem 2.1, there exists $v_{0} \in K_{2}$ such that $v_{0}=G_{2}\left(v_{0}\right)$. To finish the proof, it is sufficient to take $u_{0}=\Phi_{2}\left(v_{0}\right)$.

In a similar way to the previous proofs, by making use of the operator

$$
\Phi_{3}(v)(x)=(1-x) \int_{0}^{x} v(s) d s+\int_{x}^{1}(1-s) v(s) d s+b x+a-b,
$$

we obtain the following

Theorem 3.3. Let $f:[0,1] \times(0, \infty) \times(-\infty, 0) \rightarrow \mathbb{R}$. Assume that

(a) $x \rightarrow f(x, y, z)$ is measurable for every $(y, z) \in(0, \infty) \times(-\infty, 0)$;

(b) $(y, z) \rightarrow f(x, y, z)$ is continuous for almost every $x \in(0,1)$;

(c) there exists a function $\eta:[0,1] \rightarrow \mathbb{R}$, with $\eta(x) \geq 0$ for almost every $x \in(0,1)$, and a constant $r>0$ such that

$\left(\mathrm{c}_{1}\right) e_{\eta, r}(x)>\eta(x)$ for almost every $x \in(0,1)$;

$\left(\mathrm{c}_{2}\right) E_{\eta, r} \in L^{1}([0,1])$

$\left(\mathrm{c}_{3}\right)\left\|E_{\eta, r}\right\|_{1} \leq r$.

Then problem $\left(\mathrm{P}_{3}\right)$ admits at least one generalized solution $u \in W^{2,1}([0,1])$ such that $u(x)>0$ for every $x \in[0,1)$.

Remark 3.1. If $\eta \equiv 0$ the set $V$ becomes

$$
\begin{aligned}
V \equiv V(r, x)=\{(y, z) \in(0, \infty) \times(0, \infty): \\
b x+a \leq y \leq r x+b x+a ; b \leq z \leq b+r\}
\end{aligned}
$$

and condition (c) of Theorem 3.1 takes the following form:

(c) there exists a constant $r>0$ such that

$\left(c_{1}\right) m_{r}(x)=\inf _{V} f(x, \cdot, \cdot)>0$ for almost every $x \in(0,1)$;

$\left(c_{2}\right)$ the function $M_{r}(x)=\sup _{V} f(x, \cdot, \cdot)$ belongs to $L^{1}([0,1])$;

$\left(c_{3}\right)\left\|M_{r}\right\|_{1} \leq r$.

Likewise, conditions (c) of Theorems 3.2 and 3.3 become

(c) there exists a constant $r>0$ such that

$\left(\mathrm{c}_{1}\right) l_{r}(x)=\inf _{T} f(x, \cdot, \cdot)>0$ for almost every $x \in(0,1)$;

$\left(c_{2}\right)$ the function $L_{r}(x)=\sup _{T} f(x, \cdot, \cdot)$ belongs to $L^{1}([0,1])$;

$\left(\mathrm{c}_{3}\right)\left\|L_{r}\right\|_{1} \leq r$, 
where

$$
\begin{aligned}
T \equiv T(r, x)=\{(y, z) \in(0, \infty) \times(-\infty, \infty) \backslash\{0\}: \\
(b-a) x+a \leq y \leq r x(x-1)+(b-a) x+a ; \\
(b-a) \leq z \leq 2 r+(b-a)\},
\end{aligned}
$$

and

(c) there exists a constant $r>0$ such that

$\left(\mathrm{c}_{1}\right) e_{r}(x)=\inf _{S} f(x, \cdot, \cdot)>0$ for almost every $x \in(0,1)$;

$\left(c_{2}\right)$ the function $E_{r}(x)=\sup _{S} f(x, \cdot, \cdot)$ belongs to $L^{1}([0,1])$;

$\left(\mathrm{c}_{3}\right)\left\|E_{r}\right\|_{1} \leq r$,

where

$$
\begin{aligned}
& S \equiv S(r, x)=\{(y, z) \in(0, \infty) \times(-\infty, 0): \\
&b x+a-b \leq y \leq r(1-x)+b x+a-b ;-b \leq-z \leq-b+r\} .
\end{aligned}
$$

In this context, we note that Theorem 3.2 extends Theorem 1 of [4].

We point out that, instead of the set $V$ [resp. $T$ or $S]$, an appropriate set that contains $V$ [resp. $T$ or $S$ ] (for instance, $\{(y, z) \in(0, \infty) \times(0, \infty)$ : $b x+a \leq y \leq r+b+a ; b \leq z \leq b+r\} \supseteq V$ if $b>0$, or $\{(y, z) \in$ $(0, \infty) \times(-\infty, \infty) \backslash\{0\}: a \leq y \leq r+a+b ;(b-a) \leq z \leq 2 r+(b-a)\} \supseteq T$ if $b>a>0$ ) can be considered, and so the previous theorems take a more practical and simpler form. Theorem 1 of [4] is an example; we now give two other examples.

Theorem 3.4. Let $f:[0,1] \times(0, \infty) \rightarrow \mathbb{R}$ be such that

(i) $x \rightarrow f(x, y)$ is measurable for every $y \in(0, \infty)$;

(ii) $y \rightarrow f(x, y)$ is continuous for almost every $x \in(0,1)$;

(iii) there exists a constant $r>0$ such that

$$
\inf _{a \leq y \leq a+b+r} f(x, y)>0 \quad \text { for almost every } x \in(0,1),
$$

and the function $x \rightarrow \sup _{a \leq y \leq a+b+r} f(x, y)$ belongs to $L^{1}([0,1])$ and has norm less than or equal to $r$.

Then the problem

$$
y^{\prime \prime}+f(x, y)=0, \quad y(0)=a>0, \quad y^{\prime}(1)=b \geq 0,
$$

admits at least one generalized solution $u \in W^{2,1}([0,1])$ such that $u(x)>0$ for every $x \in[0,1]$.

Proof. It is sufficient to observe that

$$
\begin{aligned}
V & =\{y \in(0, \infty): b x+a \leq y \leq r x+b x+a\} \\
& \subseteq\{y \in(0, \infty): a \leq y \leq a+b+r\} .
\end{aligned}
$$

Then, by Remark 3.1, assumption (c) of Theorem 3.1 holds. 
Theorem 3.5. Let $f:[0,1] \times(0, \infty) \rightarrow \mathbb{R}$ satisfy (i) and (ii) of Theorem 3.4. Further, assume that

(iii) there exists a constant $r>0$ such that

$$
\inf _{(1-x) a \leq y \leq r+a+b} f(x, y)>0 \quad \text { for almost every } x \in(0,1),
$$

and the function $x \rightarrow \sup _{(1-x) a \leq y \leq r+a+b} f(x, y)$ belongs to $L^{1}([0,1])$ and has norm less than or equal to $r$.

Then the problem

$$
y^{\prime \prime}+f(x, y)=0, \quad y(0)=a>0, \quad y(1)=b \geq 0,
$$

admits at least one generalized solution $u \in W^{2,1}([0,1])$ such that $u(x)>0$ for every $x \in[0,1]$.

Proof. It is sufficient to observe that

$$
\begin{aligned}
T & =\{y \in(0, \infty):(b-a) x+a \leq y \leq r x(1-x)+(b-a) x+a\} \\
& \subseteq\{y \in(0, \infty):(1-x) a+b \leq y \leq r+a+b\} .
\end{aligned}
$$

Re mark 3.2. When $f$ is continuous every generalized solution to problem $\left(\mathrm{P}_{1}\right),\left(\mathrm{P}_{2}\right)$ or $\left(\mathrm{P}_{3}\right)$ is classical.

If $f$ is continuous in $(0,1) \times(0, \infty) \times(0, \infty)$ the solution $u$ to $\left(\mathrm{P}_{1}\right)$ belongs to $C^{1}([0,1]) \cap C^{2}((0,1))$ and $u^{\prime \prime}(x)=f\left(x, u(x), u^{\prime}(x)\right)$ for every $x \in(0,1)$; if $f$ is continuous in $(0,1) \times(0, \infty) \times(-\infty, \infty) \backslash\{0\}$ any solution $u$ to $\left(\mathrm{P}_{2}\right)$ belongs to $C^{1}([0,1]) \cap C^{2}((0,1))$ and $u^{\prime \prime}(x)=f\left(x, u(x), u^{\prime}(x)\right)$ for every $x \in(0,1)$.

Similar arguments hold for problem $\left(\mathrm{P}_{3}\right)$.

Remark 3.3. We emphasize that in the previous theorems it is not required that the function $(x, y, z) \rightarrow f(x, y, z)$ be nonincreasing in $y$ and/or $z$ (compare with $[7,9,10]$ ). Hence the singularities at $y=0$ and/or $z=0$ are not necessarily imposed by the conditions $\lim _{y \rightarrow 0^{+}} f(x, y, z)=\infty$ for every $x$ and $z$ and/or (in problem $\left(\mathrm{P}_{1}\right)$ or $\left.\left(\mathrm{P}_{3}\right)\right) \lim _{z \rightarrow 0^{+}} f(x, y, z)=\infty$ for every $x$ and $y$. Thus, we need not suppose, for instance, $\lim _{y \rightarrow 0^{+}} f(x, y)=\infty$ for every $x$ (as for example in Theorem 2.2 of [7]), even if $f$ has a singularity at $y=0$. The problem $\left(\mathrm{P}_{2}\right)$ in Remark 3 of [4], namely

$$
y^{\prime \prime}+x\left[|\sin 1 / y|^{1 / 2}+|y|^{1 / 2}+x\right]=0, \quad y(0)=0, \quad y(1)=b>0,
$$

has $f$ that does not satisfy this condition, but admits at least one positive solution owing to Theorem 3.2.

Moreover, we want to point out, as regards problem $\left(\mathrm{P}_{2}\right)$, that our theorem can be applied when $f$ has a singularity at $z=0$ of the type

$$
\lim _{z \rightarrow 0^{+}} f(x, y, z) \neq \lim _{z \rightarrow 0^{-}} f(x, y, z)
$$

for every $x \in[0,1]$ and every $y \in(0, \infty)$ (see the example in Remark 4.4). 
On the other hand, our theorems can also be used if $f$ is nonincreasing in $y$ and/or $z$, as will be seen in Section 4 .

R e mark 3.4. Theorems 3.1-3.3 extend and improve some theorems of [10] (hence of [9]); moreover, they imply existence theorems independent of some of those of [10]. In Section 4 we will develop this argument.

4. Some special cases. In this section we examine some consequences of Theorems 3.1-3.3. We give conditions that imply assumption (c) of the previous theorems. In some cases we improve the results of [10], and in some cases we obtain independent results.

Let $h:[0,1] \times(0, \infty) \times(0, \infty) \rightarrow \mathbb{R}$ be continuous and let $\psi$ be a function such that $1 / \psi:[0,1] \rightarrow \mathbb{R}$ is continuous with $\psi>0$ in $(0,1)$. Taking into account Remark 3.2, from Theorem 3.1 we have the following

\section{Theorem 4.1. Assume that}

(1) There exists $\eta:[0,1] \rightarrow \mathbb{R}$ continuous with $\eta(x)>0$ for every $x \in$ $[0,1]$ such that $h(x, y, z) \geq \eta(x)$ for every $x \in[0,1]$ and $(y, z) \in$ $(0, \infty) \times(0, \infty)$.

(2) There exist $g$ and $\phi$ continuous, positive and nonincreasing in $(0, \infty)$ such that $0<h(x, y, z) \leq g(y) \phi(z)$ for every $x \in[0,1]$ and $(y, z) \in$ $(0, \infty) \times(0, \infty)$.

$$
\begin{aligned}
\int_{0}^{1} \psi(x) g\left(\int_{0}^{x} s \psi(s) \eta(s) d s+x \int_{x}^{1} \psi(s) \eta(s)\right. & \\
& \times \phi\left(\int_{x}^{1} \psi(s) \eta(s) d s\right) d x<\infty .
\end{aligned}
$$

Then the problem

$$
y^{\prime \prime}+\psi(x) h\left(x, y, y^{\prime}\right)=0, \quad y(0)=a=0, \quad y^{\prime}(1)=b=0,
$$

admits at least one solution $u \in C^{1}([0,1]) \cap C^{2}((0,1))$ such that $u(x)>0$ for every $x \in(0,1]$.

Proof. By choosing

$$
r=\int_{0}^{1} \psi(x) g\left(\int_{0}^{x} s \psi(s) \eta(s) d s+x \int_{x}^{1} \psi(s) \eta(s) d s\right) \phi\left(\int_{x}^{1} \psi(s) \eta(s) d s\right) d x
$$

we obtain

$$
\inf _{V(\psi \eta, x, r)} \psi(x) h(x, y, z)>\psi(x) \eta(x)
$$


and

$$
\begin{aligned}
& \int_{0}^{1} \sup _{V(\psi \eta, x, r)} \psi(x) h(x, y, z) d x \\
& \quad \leq \int_{0}^{1} \psi(x) g\left(\int_{0}^{x} s \psi(s) \eta(s) d s+x \int_{x}^{1} \psi(s) \eta(s) d s\right) \phi\left(\int_{x}^{1} \psi(s) \eta(s) d s\right) d x=r .
\end{aligned}
$$

Hence, from Theorem 3.1 we obtain the conclusion.

R e mark 4.1. If $a \neq 0$ and $b \neq 0$, instead of (1) and (3), we can assume

$$
\int_{0}^{1} \psi(x) d x<\infty .
$$

Indeed, we choose $r=g(a) \phi(b)\|\psi\|_{1}$ and apply Remark 3.1 and Theorem 3.1. In this case we have thus obtained Theorem 2.2 of [10].

If $b \neq 0$ and $a=0$ we can substitute (1) and (3) with the condition

$$
\int_{0}^{1} \psi(x) g(b x) d x<\infty .
$$

Indeed, we choose $r=\phi(b) \int_{0}^{1} \psi(x) g(b x) d x$. In this case we have improved Theorem 4.1 of [10] because condition $\left(3^{\prime \prime}\right)$ is more general than

(A) There exists $p>1$ such that $\int_{0}^{1} g^{p}(b x) \psi^{p}(x) d x<\infty$.

Also if $h$ is independent of $z$, we can substitute (1) and (3) of Theorem 4.1 with $\left(3^{\prime \prime}\right)$ and find that the problem

$$
y^{\prime \prime}+\psi(x) h(x, y)=0, \quad y(0)=0, \quad y^{\prime}(1)=b>0,
$$

admits at least one solution $u \in C^{1}([0,1]) \cap C^{2}((0,1))$ such that $u(x)>0$ for every $x \in(0,1]$. In this case we have improved one of the cases considered in Theorem 3.1 of [10] because the condition $\left(3^{\prime \prime}\right)$ is more general than

(B) There exists $p>1$ such that $\psi \in L^{p}([0,1])$ and $g \in L^{p /(p-1)}([0,1])$, as is easily seen from the Hölder inequality.

As an example we observe that in the following problem we can apply our results but it is not possible to apply Theorem 3.1 of [10] (cf. also Theorem 2.4 of [9]):

$$
y^{\prime \prime}+(x / y)^{2}=0, \quad y(0)=0, \quad y^{\prime}(1)=1 .
$$

When $f$ is not nonincreasing in $y$, from Theorem 3.1 we obtain some existence results with hypotheses different from those of Theorems 5.4 and 5.5 of [10]. For example, we have 
TheOrem 4.2. Assume that

(1) There exists $\phi$ continuous, positive and nonincreasing in $(0, \infty)$ and there exists $g$ continuous in $[a, \infty)$ such that $0<h(x, y, z) \leq g(y) \phi(z)$ for every $x \in[0,1]$ and $(y, z) \in(a, \infty) \times(0, \infty)$.

(2) There exists $r>0$ such that

$$
\sup _{[a, a+b+r]} g(y) \leq r / \phi(b) .
$$

Then the problem

$$
y^{\prime \prime}+h\left(x, y, y^{\prime}\right)=0, \quad y(0)=a \geq 0, \quad y^{\prime}(1)=b>0,
$$

admits at least one solution $u \in C^{2}([0,1])$ such that $u(x)>0$ for every $x \in(0,1]$.

Pr o of. One has $V \subseteq\{(y, z) \in(0, \infty) \times(0, \infty): b x+a \leq y \leq r+b+a ; b \leq$ $z \leq b+r\}$. Hence, $\inf _{V} h(x, y, z)>0$ for every $x \in[0,1]$ and

$$
\begin{aligned}
\int_{0}^{1} \sup _{V} h(x, y, z) d x & \leq \int_{0}^{1} \sup _{[a, a+b+r]} g(y) \sup _{[b, b+r]} \phi(z) d x \\
& =\phi(b) \sup _{[a, a+b+r]} g(y) \leq r .
\end{aligned}
$$

Then, from Theorem 3.1, we obtain the conclusion.

Remark 4.2. If $b=0$ we must substitute (2) of Theorem 4.2 with

$\left(2^{\prime}\right) \quad$ There exist $r>0$ and $\eta:[0,1] \rightarrow \mathbb{R}$, with $\eta(x)>0$ and continuous in $[0,1]$, such that $h(x, y, z) \geq \eta(x)$ for every $x \in[0,1]$ and $(y, z) \in$ $(a, a+r] \times(0, r]$, and

$$
\sup _{[a, a+r]} g(y) \leq r / \int_{0}^{1} \phi\left(\int_{x}^{1} \eta(s) d s\right) d x .
$$

Remark 4.3. Theorem 4.2 and Remark 4.2 cover some cases that do not satisfy the assumption

(C) There exist $A \geq 0, B \geq 0,0 \leq q<1$, such that

$$
\int_{a}^{z} g(u) d u \leq \int_{0}^{A z^{q}+B} \frac{u}{\phi(u)} d u \quad \text { for all } z \in[a, \infty),
$$

required in Theorems 5.4 and 5.5 of [10].

We give two examples. The problem

$$
y^{\prime \prime}+y / y^{\prime}=0, \quad y(0)=0, \quad y^{\prime}(1)=2,
$$

owing to Theorem 4.2, admits at least one positive solution $u \in C^{2}([0,1])$, while, as is easy to see, the functions $y \rightarrow g(y)=y$ and $z \rightarrow \phi(z)=1 / z$ do not satisfy the condition (C). 
The problem

$$
y^{\prime \prime}+\sqrt[4]{y+1} / \sqrt{y^{\prime}}=0, \quad y(0)=0, \quad y^{\prime}(1)=0,
$$

owing to Remark 4.2, admits at least one positive solution $u \in C^{1}([0,1]) \cap$ $C^{2}([0,1))$, while, as is easy to see, the functions $y \rightarrow g(y)=\sqrt[4]{y+1}$ and $z \rightarrow \phi(z)=1 / \sqrt{z}$ do not satisfy the condition (C).

Now we show that Theorem 3.2 extends and improves Theorem 2.3 of $[10]$ and most cases of Theorem 3.2 of [10]. Let $h:[0,1] \times(0, \infty) \times(-\infty, \infty) \backslash\{0\} \rightarrow$ $\mathbb{R}$, and $1 / \psi:[0,1] \rightarrow \mathbb{R}$ a continuous function such that $\psi$ is positive in $(0,1)$. We have

Theorem 4.3 ([10, Theorem 2.3]). Assume that

(1) There exist $g$ and $\phi$ continuous, positive and nonincreasing in $(0, \infty)$ and $[0, \infty)$ respectively such that $0<h(x, y, z) \leq g(y) \phi(|z|)$ for every $x \in[0,1]$ and $(y, z) \in(0, \infty) \times(-\infty, \infty)$.

$$
\int_{0}^{1} \psi(x) d x<\infty .
$$

Then the problem

$$
y^{\prime \prime}+\psi(x) h\left(x, y, y^{\prime}\right)=0, \quad y(0)=a>0, \quad y(1)=b>0,
$$

admits at least one solution $u \in C^{1}([0,1]) \cap C^{2}((0,1))$ such that $u(x)>0$ for every $x \in[0,1]$.

Proof. We choose $r=\phi(0) g(\min \{a, b\})\|\psi\|_{1}$ and, taking into account

$$
\begin{aligned}
T \subseteq\{(y, z) & \in(0, \infty) \times(-\infty, \infty) \backslash\{0\}: \\
& \min \{a, b\} \leq y \leq r+a+(b-a) x ;(b-a) \leq z \leq 2 r+(b-a)\},
\end{aligned}
$$

we obtain $\int_{0}^{1} \sup _{T} \psi(x) h(x, y, z) d x \leq \phi(0) g(\min \{a, b\})\|\psi\|_{1}=r$. From Theorem 3.2, taking into account Remarks 3.1 and 3.2, we have the conclusion.

R e mark 4.4. If $b>a$ we can substitute assumption (1) of Theorem 4.3 with the more general assumption

$\left(1^{\prime}\right)$ There exist $g$ and $\phi$ continuous, positive and nonincreasing in $(0, \infty)$ such that $0<h(x, y, z) \leq g(y) \phi(z)$ for every $x \in[0,1]$ and $(y, z) \in$ $(0, \infty) \times(0, \infty)$.

In this case we choose $r=\phi(b-a) g(a)\|\psi\|_{1}$.

As an example, the problem

$$
y^{\prime \prime}+\frac{1}{\sqrt{x} y y^{\prime}}=0, \quad y(0)=1, \quad y(1)=2,
$$


admits at least one solution $u \in C^{1}([0,1]) \cap C^{2}((0,1))$, but the functions $y \rightarrow g(y)=1 / y$ and $z \rightarrow \phi(z)=1 / z$ do not satisfy assumption (1) of Theorem 4.3 .

If $b \leq a$, instead of (1), it is sufficient to assume

$\left(1^{\prime \prime}\right) \quad$ There exists $g$ continuous, positive and nonincreasing in $(0, \infty)$ and there exists $\phi$ continuous in $(-\infty, \infty) \backslash\{0\}$ such that $0<h(x, y, z) \leq$ $g(y) \phi(z)$ for every $x \in[0,1]$ and $(y, z) \in(0, \infty) \times(-\infty, \infty)$, and

$$
\sup _{[b-a, \infty)} \phi(z)<\infty
$$

by choosing $r=\sup _{[b-a, \infty)} \phi(z) g(\min \{a, b\})\|\psi\|_{1}$.

In this case it is possible to consider a singularity at $z=0$ of the type

$$
\lim _{z \rightarrow 0^{+}} h(x, y, z) \neq \lim _{z \rightarrow 0^{-}} h(x, y, z) .
$$

For instance, the problem

$$
\left\{\begin{array}{l}
y^{\prime \prime}+\frac{1}{\sqrt{x}} \frac{1}{y} \phi\left(y^{\prime}\right)=0, \\
y(0)=2, \quad y(1)=1,
\end{array} \quad \text { where } \quad \phi(z)= \begin{cases}1 & \text { if } z \geq 0, \\
1 / 2 & \text { if } z<0,\end{cases}\right.
$$

admits at least one positive solution $u \in C^{1}([0,1]) \cap C^{2}((0,1))$.

The previous results show that Theorem 3.2 improves Theorem 2.3 of $[10]$.

Again from Theorem 3.2 we obtain the following

Theorem 4.4. Assume that

(1) There exists $g$ continuous and nonincreasing in $(0, \infty)$ such that $0<$ $h(x, y) \leq g(y)$ for every $(x, y) \in[0,1] \times(0, \infty)$.

$$
\int_{0}^{1} \psi(x) g((1-x) a) d x<\infty \text {. }
$$

Then the problem

$$
y^{\prime \prime}+\psi(x) h(x, y)=0, \quad y(0)=a>0, \quad y(1)=0,
$$

admits at least one solution $u \in C^{1}([0,1]) \cap C^{2}((0,1))$ such that $u(x)>0$ for every $x \in[0,1)$.

Proof. We choose $r=\int_{0}^{1} \psi(x) g((1-x) a) d x$ and observe that

$$
\int_{0}^{1} \sup _{T} \psi(x) h(x, y) d x \leq \int_{0}^{1} \psi(x) g((1-x) a) d x=r .
$$

From Theorem 3.2 and Remarks 3.1 and 3.2 we obtain the conclusion. 
Re mark 4.5. Theorem 4.4 improves Theorem 3.2 of [10] (when $a>0$ ) because condition (2) is more general than

(B) There exists $p>1$ such that $\psi \in L^{p}([0,1])$ and $g \in L^{p /(p-1)}([0,1])$, required by [10]. In fact, if (B) holds, then (2) follows by the Hölder inequality, but not conversely, as is easily shown by the function $\psi(x) h(x, y)=$ $a(1-x) / y$.

The case $a=0$ and $b \neq 0$ has already been considered in [4].

If $a=b=0$ from Theorem 3.2 we obtain the following

Theorem 4.5. Assume that

(1) There exists $\eta$ positive and continuous in $[0,1]$ such that $h(x, y) \geq$ $\eta(x)$ for every $(x, y) \in[0,1] \times(0, \infty)$.

(2) There exists $g$ continuous and nonincreasing in $(0, \infty)$ such that $0<$ $h(x, y) \leq g(y)$ for every $(x, y) \in[0,1] \times(0, \infty)$.

$$
\int_{0}^{1} \psi(x) g\left((1-x) \int_{0}^{x} t \eta(t) \psi(t) d t+x \int_{x}^{1}(1-t) \eta(t) \psi(t) d t\right) d x<\infty .
$$

Then the problem

$$
y^{\prime \prime}+\psi(x) h(x, y)=0, \quad y(0)=0, \quad y(1)=0,
$$

admits at least one solution $u \in C^{1}([0,1]) \cap C^{2}((0,1))$ such that $u(x)>0$ for every $x \in(0,1)$.

Proof. By choosing

$$
r=\int_{0}^{1} \psi(x) g\left((1-x) \int_{0}^{x} t \eta(t) \psi(t) d t+x \int_{x}^{1}(1-t) \eta(t) \psi(t) d t\right) d x
$$

we have $\inf _{T(\eta \psi, r, x)} \psi(x) h(x, y) \geq \eta(x) \psi(x)$ and

$$
\int_{0}^{1} \sup _{T(\eta \psi, r, x)} \psi(x) h(x, y) d x \leq \int_{0}^{1} \psi(x) \sup _{T(\eta \psi, r, x)} g(y) d x \leq r .
$$

From Theorem 3.2 we obtain the conclusion.

Remark 4.6. In a similar way it is possible to show that Theorem 3.3 extends and improves some of the results of [10] as regards Problem $\left(\mathrm{P}_{3}\right)$. As an example, we give the following theorem (compare with Remark on p. 236 of [10]).

\section{Theorem 4.6. Assume that}

(1) There exists $g$ continuous and nonincreasing in $(0, \infty)$ such that $0<$ $h(x, y) \leq g(y)$ for every $(x, y) \in[0,1] \times(0, \infty)$. 


$$
\int_{0}^{1} \psi(x) g((1-x) b) d x<\infty
$$

Then the problem

$$
y^{\prime \prime}+\psi(x) h(x, y)=0, \quad y(1)=0, \quad y^{\prime}(0)=b<0
$$

admits at least one solution $u \in C^{1}([0,1]) \cap C^{2}((0,1))$ such that $u(x)>0$ for every $x \in[0,1)$.

Proof. It is sufficient to choose $r=\int_{0}^{1} \psi(x) g((1-x) b) d x$ and from Theorem 3.3 we obtain the conclusion.

\section{References}

[1] O. Arino, S. Gautier and J. P. Penot, A fixed point theorem for sequentially continuous mappings with application to ordinary differential equations, Funkcial. Ekvac. 27 (1984), 273-279.

[2] L. E. Bobisud, Existence of positive solutions to some nonlinear singular boundary value problems on finite and infinite intervals, J. Math. Anal. Appl. 173 (1993), 6983.

[3] L. E. Bobisud, D. O'Regan and W. D. Royalty, Solvability of some nonlinear boundary value problems, Nonlinear Anal. 12 (1988), 855-869.

[4] G. Bonanno, An existence theorem of positive solutions to a singular nonlinear boundary value problem, Comment. Math. Univ. Carolin. 36 (1995), 609-614.

[5] A. Callegari and A. Nachman, Some singular, nonlinear differential equations arising in boundary layer theory, J. Math. Anal. Appl. 64 (1978), 96-105.

[6] J. Diestel and J. J. Uhl, Jr., Vector Measures, Math. Surveys 15, Amer. Math. Soc., 1977.

[7] J. A. Gatica, V. Oliker and P. Waltman, Singular nonlinear boundary value problems for second-order ordinary differential equations, J. Differential Equations 79 (1989), 62-78.

[8] A. Nachman and A. Callegari, A nonlinear singular boundary value problem in theory of pseudoplastic fluids, SIAM J. Appl. Math. 38 (1980), 275-281.

[9] D. O'Regan, Positive solutions to singular and nonsingular second order boundary value problems, J. Math. Anal. Appl. 142 (1989), 40-52.

[10] - Existence of positive solutions to some singular and nonsingular second order boundary value problems, J. Differential Equations 84 (1990), 228-251.

[11] S. D. Taliaferro, A nonlinear singular boundary value problem, Nonlinear Anal. 3 (1979), 897-904.

Dipartimento di Ingegneria Elettronica e Matematica Applicata Università di Reggio Calabria

via E. Cuzzocrea 48

89128 Reggio Calabria, Italy 